\title{
Pregnancy with Hypertrophic Cardiomyopathy - A Potential Risk Factor for Maternal Mortality
}

\section{Kumare BD, Kawthalkar A}

Department of Obstetrics and Gynecology

NKP Salve Institute of Medical Sciences and Research Centre, Digdoh Hills, Higna Road

Nagpur, Maharashtra, India

\section{Corresponding Author}

Bhavna D Kumare

Department of Obstetrics and Gynecology

NKP Salve Institute of Medical Sciences and Research Centre, Digdoh Hills, Higna Road

Nagpur, Maharashtra, India

E-mail: bhavnakumare@yahoo.co.in

\section{Citation}

Kumare BD, Kawthalkar A. Pregnancy with

Hypertrophic Cardiomyopathy - A potential Risk

Factor for Maternal Mortality. Kathmandu Univ Med J 2013;44(4):335-337.

\begin{abstract}
We report a case of a 21 year old married woman diagnosed to have severe hypertrophic cardiomyopathy at 12 weeks of gestation by echocardiograph at Lata Mangeshkar Hospital, Nagpur on $12^{\text {th }}$ June, 2012. Her mother's and maternal uncle's sudden death at young age along with severe form of disease brought a lot of apprehension in the beginning considering the possible catastrophic effect of hemodynamic changes associated with pregnancy and labor. She was asymptomatic before pregnancy but developed dyspnoea on exertion during pregnancy and hence was started on calcium channel blocker verapamil. She remained asymptomatic throughout pregnancy and later underwent elective caesarean section at term under epidural anaesthesia for major cephalopelvic disproportion with uneventful postoperative course. Current data on pregnancy outcome in Hypertrophic cardiomyopathy are limited in Southasian countries despite its high prevalence in general population. This case report illustrates that with favourable clinical profile and specialist multidisciplinary team management women with hypertrophic cardiomyopathy tolerate pregnancy well with good outcome.
\end{abstract}

\section{KEY WORDS}

Hypertrophic cardiomyopathy, HCM, pregnancy

\section{INTRODUCTION}

Hypertrophic cardiomyopathy ( $\mathrm{HCM}$ ) is a unique inherited heart disease which is characterized by primary hypertrophy of a non-dilated ventricle. It is not a rare disease but that it has many disguise. ${ }^{1}$ The phenotypic expression is very heterogeneous both in terms of age and severity leading to variety of clinical presentation. ${ }^{2}$ The clinical course can range from a lifelong asymptomatic state to one where individuals are highly symptomatic with heart failure and arrhythmias. ${ }^{2}$ Though the highest incidence of the disease is found in young adults, only a limited number of pregnancies in women with HCM have been described in the litearture. ${ }^{3}$ Most of the cases and studies reported in literature are from European and American population however; HCM appears in all racial groups. We report a successful outcome of HCM during pregnancy in an Indian woman who was managed by expert multidisciplinary team at tertiary care centre.

\section{CASE-REPORT}

A 21 year old married woman was first seen in ANC clinic at 12 weeks of pregnancy with abnormal cardiac signs at Lata
Mangeshkar hospital, Nagpur on $12^{\text {th }}$ June 2012 . This was her second pregnancy, the first having occurred in 2008 at the age of 20 year which ended in a spontaneous abortion not requiring surgical evacuation. She was symptomatic on moderate exercise (NYHA -grade 2) in present pregnancy. There was no history of Rheumatic fever in past but there was history of sudden death of her mother during her delivery and of maternal uncle at the young age of 40. Patient was from lower socioeconomic class from rural India with no family support and hence was never evaluated before pregnancy.

On examination, she had grade $4 / 6$ pansystolic murmur at mitral and pulmonary area radiating to left axilla and back, Her arterial pulse, Blood pressure, JVP, first and second heart sounds were normal. Respiratory system was normal. Pelvic examination confirmed 12 weeks pregnancy.

Her 2DEcho revealed severe concentric left ventricular hypertrophy with moderate mitral regurgitation with HCM with diastolic dysfunction. Left ventricular ejection fraction was $55-60 \%$. The ECG showed sinus tachycardia with $T$ wave inversion suggestive of Left ventricular strain. 
The diagnosis of Hypertrophic cardiomyopathy of severe variety with moderate mitral regurgitation was made. Foetal echocardiography at 22 weeks of gestation was normal.

She was started on calcium channel blocker Verapamil $80 \mathrm{mg}$ thrice a day from 14 weeks of pregnancy as she developed dyspnoea on exertion during pregnancy. She had syncopal attacks in squatting position in mid-trimester so she was advised to use commode instead of Indian toilets. She remained asymptomatic for the rest of her pregnancy. She was admitted at 37 weeks with major degree of cepahalopelvic disproportion and underwent Elective C-section under antibiotic prophylaxis in epidural anaesthesia. Central venous pressure line was placed. The baby delivered was a live female baby of 2700 grams with no evidence of heart disease at birth. She received slow Oxytocin infusion after the delivery of the baby. Her intraoperative and postoperative period was uneventful. She was advised injectable progesterone for contraception and was discharged in good condition after physician consultation on oral metoprolol $25 \mathrm{mg}$ once a day.

\section{DISCUSSION}

HCM has recently been defined by the European Society of Cardiology (ESC) as increased ventricular wall thickness or mass in the absence of loading conditions (hypertension, valve disease) sufficient to cause observed abnormality. ${ }^{4}$ It has an autosomal dominant inheritance with variable penetrance. ${ }^{5}$ Around $70 \%$ of $\mathrm{HCM}$ patients have at least 1 affected family member, but sporadic mutations are also known to occur. ${ }^{4}$ The estimated prevalence of HCM is 1 in 500 but many individuals have a subclinical phenotype and it often goes undiagnosed in the community. ${ }^{6}$ Occult $\mathrm{HCM}$ may manifest for first time during pregnancy. HCM is increasingly being diagnosed in pregnant women because of widespread use of 2D echocardiography. Left ventricular outflow tract obstruction and mitral regurgitation are two principal hemodynamic concerns.

The published data on pregnancy in HCM are scarce and contain some inconsistencies. The data that are available demonstrate that the pregnancy is well tolerated, however a subset of patients do experience significant complications. ${ }^{3} \mathrm{HCM}$ is considered a WHO class II or III risk lesion, implying there is a moderate risk of morbidity in most women and potentially a significant risk for some. ${ }^{7}$ The diagnosis of HCM among asymptomatic women is not considered a contraindication for pregnancy, but patients should be carefully evaluated in regard to the risk of pregnancy. For patients (mother or father) with HCM, preconception genetic counselling is indicated. ${ }^{7}$ Transmission of heart disease to offspring should be discussed as the risk is approximately $50 \%$ compared to background risk of any congenital heart disease of one percent. The risk of pregnancy is very much dependent on pre-pregnancy clinical status. ${ }^{2}$ Asymptomatic women generally do well through pregnancy but symptomatic women; those with history arrhythmias, significant LVOT obstruction, left ventricular systolic dysfunction are prone for complications (pulmonary oedema, arrhythmia and decline to NYHA III-IV) during pregnancy. ${ }^{2}$ Pre-conception risk assessment of HCM should be done using modified WHO classification as per the ESC guidelines for the management of cardiovascular diseases during pregnancy. ${ }^{7}$ Women with HCM should have frequent evaluation during pregnancy and should be managed at tertiary care centre by a multidisciplinary team. ${ }^{7}$

Women taking beta-blockers prior to pregnancy should continue them during pregnancy and any women who become symptomatic during pregnancy should start betablockers along with diuretics if required. ${ }^{7}$ Metoprolol is the preferred beta-blocker for control of atrial fibrillation because of ample experience of its use in pregnancy. ${ }^{7}$ Infants should be monitored for bradycardia and hypoglycaemia following birth. ${ }^{7}$ Atenolol is best avoided due to its risk of foetal growth restriction. ${ }^{7}$ Verapamil can be used in pregnancy if woman is not responding to beta-blockers or there are contraindications to use of beta-blockers but caution is necessary due to risk of foetal atrioventricular block. ${ }^{7}$ Foetal 2D echo should be offered at 20 weeks of pregnancy. Vaginal delivery is recommended unless there are obstetric indications for caesarean delivery. ${ }^{7}$ Good pain management of labor and instrumental delivery in second stage will minimize maternal cardiac stress. Regional anaesthesia for caesarean section should be carefully administered to avoid hypotension as vasodilatation is poorly tolerated and single shot spinal anaesthesia should be avoided. ${ }^{2}$ Invasive monitoring is best avoided due to increased risk of arrhythmias and thromboembolism. ${ }^{7}$ Non-invasive monitoring with $2 \mathrm{D}$ echo and continuous ECG can be effectively accomplished when needed. ${ }^{8}$ Oxytocin can induce vasodilatation so it should only be given as slow intravenous infusion. ${ }^{2}$ The dose should be kept to minimum (5 units) to avoid side-effects of hypotension, tachycardia and arrhythmias. Both blood loss and fluid overload must be avoided. ${ }^{2}$ Close observation and monitoring for at least 24 hours should be done avoid the risk of pulmonary oedema due to fluid shifts associated with delivery. ${ }^{2}$

Sudden cardiac death has been the most devastating, unpredictable and recognisable complication of hypertrophic cardiomyopathy during pregnancy. ${ }^{9}$ Although only a minority of all HCM patients are susceptible to the risk of sudden death, it may be the initial manifestation of the disease in undiagnosed women. Hence, identification of this patient subset should be a priority. ${ }^{9}$ The reported total maternal mortality in women with HCM was 10 per 1000 live births which is higher as compared to general population. ${ }^{10}$ However the absolute maternal mortality is low and is confined to high risk women like those who are symptomatic before pregnancy. ${ }^{10}$

Although a family history of sudden death is generally considered an indicator of increased risk in HCM, literature 
reports that such a history is not associated with a major increase in risk. ${ }^{10}$ Our patient had positive family history of sudden death but was fortunate to have a good outcome with no complications. This could be attributed to prepregnancy asymptomatic state and/or variable phenotypic expression of the disease.

\section{CONCLUSION}

HCM is uncommon but significant cardiac lesion which remains undiagnosed in developing countries like South Asia. Care must be taken in making the diagnosis of HCM by echocardiography since systolic murmurs are quite frequent during pregnancy. Despite the potential hazards, women with HCM generally tolerate pregnancy well except for those with high risk factors. Hence identification of these high risk women is the priority that needs specialist, multidisciplinary care at tertiary centre.

\section{REFERENCES}

1. Gillian M Turner, Celia M Oakley HG Dixon. Management of pregnancy complicated by Hypertrophic Obstructive Cardiomyopathy. BMJ. 1968; 11: 281-284.

2. Pieper PG, Walker F. Pregnancy in women with hypertrophic cardiomyopathy. Neth Heart J. 2013; 21: 14-18.

3. Krul SPJ, Smagt JJ, Berg MP, Sollie KM, Pieper PG, SpaendonckZwarts KY. Systematic review of pregnancy in women with inherited cardiomyopathies. Eur J Heart Fail. 2011; 13: 584-594.

4. Elliot $P$, Anderson B, Arbustini E, Belinska Z, Cecchi F, Charron P et al. Classification of cardiomyopathies: a position statement from European Society of Cardiology working group on myocardial and pericardial disease. Eur Heart J. 2008; 29: 270-276.

5. Christiaans I, Nannenberg EA, Dooijes D, Jongbloed RJE, Postema PG, Majoor-Krakauer D et al. Founder mutations in hypertrophic cardiomyopathy in the Netherlands. Neth Heart J. 2010; 18: 248-254.

6. Mckeown PP, Muir AP. Risk assessment in hypertrophic cardiomyopathy: contemporary guidelines hampered by insufficient evidence. Heart. 2013; 99: 511-512

7. Regitz-Zagrosek V, Blomstrom LC, Borghi C, Lundquist CB, Borghi C, Cifkova $R$ et al. ESC guidelines on the management of cardiovascular diseases during pregnancy of the European Society of Cardiology. Eur Heart J. 2001; 32: 3147-3197.

8. Ferguson EA, Peach MJ, Vellman MG. Hypertrophic cardiomyopathy and caesarean section: intraoperative use of transthoracic echocardiography. Int J Obst Anesth. 2006; 15:311-316.

9. Elliott PM, Poloniecki J, Dickie S, Sharma S, Monserrat L, Varnava A, et al. Sudden death in hypertrophic cardiomyopathy: identification of high risk patients. J Am Coll Cardiol. 2000; 36: 2212-18.

10. Autore $\mathrm{C}, \mathrm{MD}$, Conte MR, Piccininno $\mathrm{M}$, Bernabo $\mathrm{P}$, Bonfiglio $\mathrm{GB}$ et al. Risk associated with pregnancy in Hypertrophic cardiomyopathy. J Am Coll Cardiol. 2002;40:1864-1869. 